\title{
Behavior of Atomic Vacancy in Puckered Arsenene
}

Lei Ao* and Zhihua Xiong*

Key Laboratory for Optoelectronics and Communication of Jiangxi Province, Jiangxi

Science \& Technology Normal University, Nanchang 330018, China.

Email: al20060608@126.com

Email: xiong_zhihua@126.com 
Table S1 Band edge locations, orbital compositions, and band gaps $(\mathrm{eV})$ of defective arsenene monolayers. The direct and indirect types of band gaps are denoted by (d) and (i), respectively.

\begin{tabular}{|c|c|c|c|c|c|c|}
\hline Type & & $\begin{array}{c}\text { VBM } \\
\text { Location }\end{array}$ & $\begin{array}{c}\text { CBM } \\
\text { Location }\end{array}$ & $\begin{array}{l}\text { VBM } \\
\text { Orbital }\end{array}$ & $\begin{array}{l}\text { CBM } \\
\text { Orbital }\end{array}$ & Band Gap \\
\hline Pristine & & $\mathrm{Y}-\Gamma$ & $\Gamma$ & $p_{\mathrm{y}}$ & $p_{\mathrm{z}}$ & 0.822 (i) \\
\hline SV-59 & $\uparrow$ & Y- $Г$ & $\Gamma$ & $p_{\mathrm{y}}$ & $p_{\mathrm{z}}$ & 0.820 (i) \\
\hline & $\downarrow$ & Y-Г & Y & $p_{\mathrm{y}}$ & $p_{\mathrm{y}}+p_{\mathrm{x}}$ & $0.336(\mathrm{i})$ \\
\hline SV-1 & $\uparrow$ & Y-Г & $\Gamma$ & $p_{\mathrm{y}}$ & $p_{\mathrm{z}}$ & 0.809 (i) \\
\hline & $\downarrow$ & Y-Г & Y & $p_{\mathrm{y}}$ & $p_{\mathrm{y}}+p_{\mathrm{x}}$ & 0.295 (i) \\
\hline SV-2 & $\uparrow$ & Y-Г & $\Gamma$ & $p_{\mathrm{y}}$ & $p_{\mathrm{y}}+p_{\mathrm{z}}+p_{\mathrm{x}}$ & 0.797 (i) \\
\hline & $\downarrow$ & $\mathrm{Y}-\Gamma$ & $\mathrm{S}$ & $p_{\mathrm{y}}$ & $p_{\mathrm{y}}+p_{\mathrm{x}}+p_{\mathrm{z}}$ & 0.093 (i) \\
\hline SV-3 & $\uparrow$ & $\Gamma-\mathrm{X}$ & $\Gamma$ & $p_{\mathrm{y}}$ & $p_{\mathrm{y}}$ & 0.508 (i) \\
\hline & $\downarrow$ & $X$ & $\Gamma$ & $p_{\mathrm{y}}$ & $p_{\mathrm{y}}+p_{\mathrm{z}}$ & $0.171(\mathrm{i})$ \\
\hline DV-585-A-1 & & $\mathrm{Y}-\Gamma$ & $\Gamma$ & $p_{\mathrm{y}}$ & $p_{\mathrm{z}}$ & 0.900 (i) \\
\hline DV-585-A-2 & & Y-Г & $\Gamma$ & $p_{\mathrm{y}}$ & $p_{\mathrm{y}}+p_{\mathrm{z}}$ & 0.897 (i) \\
\hline DV-585-A-3 & & Y-Г & $\Gamma$ & $p_{\mathrm{y}}$ & $p_{\mathrm{z}}$ & 0.901 (i) \\
\hline DV-585-B & & $\mathrm{Y}-\Gamma$ & $\mathrm{X}-\mathrm{S}$ & $p_{\mathrm{y}}$ & $p_{\mathrm{y}}+p_{\mathrm{x}}$ & 0.647 (i) \\
\hline DV-555777-1 & & $\mathrm{Y}-\Gamma$ & $\Gamma$ & $p_{\mathrm{y}}$ & $p_{\mathrm{z}}$ & 0.875 (i) \\
\hline DV-555777-2 & & Y-Г & $\Gamma$ & $p_{\mathrm{y}}$ & $p_{\mathrm{y}}+p_{\mathrm{z}}$ & 0.869 (i) \\
\hline DV-4104 & & $\mathrm{X}$ & $\Gamma$ & $p_{\mathrm{z}}$ & $p_{\mathrm{z}}$ & 0.950 (i) \\
\hline DV-5577-A-1 & & $\mathrm{Y}-\Gamma$ & $\Gamma$ & $p_{\mathrm{y}}$ & $p_{\mathrm{z}}$ & $0.956(\mathrm{i})$ \\
\hline DV-5577-A-2 & & $\mathrm{Y}-\Gamma / \Gamma$ & $\Gamma$ & $p_{\mathrm{y}} / p_{\mathrm{y}}+p_{\mathrm{z}}$ & $p_{\mathrm{z}}$ & $0.936(\mathrm{i}) / 0.941(\mathrm{~d})$ \\
\hline DV-5577-A-3 & & Y-Г & $\Gamma$ & $p_{\mathrm{y}}$ & $p_{\mathrm{z}}$ & 0.944 (i) \\
\hline DV-5577-B & & Y-Г & $\mathrm{X}$ & $p_{\mathrm{y}}$ & $p_{\mathrm{y}}+p_{\mathrm{x}}+p_{\mathrm{z}}$ & $0.584(\mathrm{i})$ \\
\hline DV-55557777-B-1 & & $\mathrm{Y}-\Gamma / \Gamma$ & $\Gamma$ & $p_{\mathrm{y}}$ & $p_{\mathrm{z}}$ & 0.945 (i)/0.951 (d) \\
\hline DV-55557777-B-2 & & $\mathrm{Y}-\Gamma / \Gamma$ & $\Gamma$ & $p_{\mathrm{y}}$ & $p_{z}$ & 0.939 (i)/0.944 (d) \\
\hline DV-55557777-B-3 & & $\Gamma$ & $\Gamma$ & $p_{\mathrm{y}}$ & $p_{\text {z }}$ & $0.940(\mathrm{~d})$ \\
\hline DV-55557777-B-4 & & $\Gamma$ & $\Gamma$ & $p_{\mathrm{y}}$ & $p_{\mathrm{z}}$ & $0.927(d)$ \\
\hline DV-55557777-A-1 & & Y-Г & $\Gamma$ & $p_{\mathrm{y}}$ & $p_{\mathrm{y}}+p_{\mathrm{z}}$ & $0.833(\mathrm{i})$ \\
\hline DV-55557777-A-2 & & Y-Г & $\Gamma$ & $p_{\mathrm{y}}$ & $p_{\mathrm{z}}$ & $0.851(\mathrm{i})$ \\
\hline DV-55557777-A-3 & & $\mathrm{Y}-\Gamma$ & $\Gamma$ & $p_{\mathrm{y}}$ & $p_{\mathrm{y}}+p_{\mathrm{z}}$ & $0.821(\mathrm{i})$ \\
\hline DV-55557777-A-4 & & $\mathrm{Y}-Г / \mathrm{Y}-Г$ & Y-Г & $p_{\mathrm{y}}$ & $p_{\mathrm{y}} / p_{\mathrm{y}}$ & 0.848 (i)/0.852 (d) \\
\hline
\end{tabular}



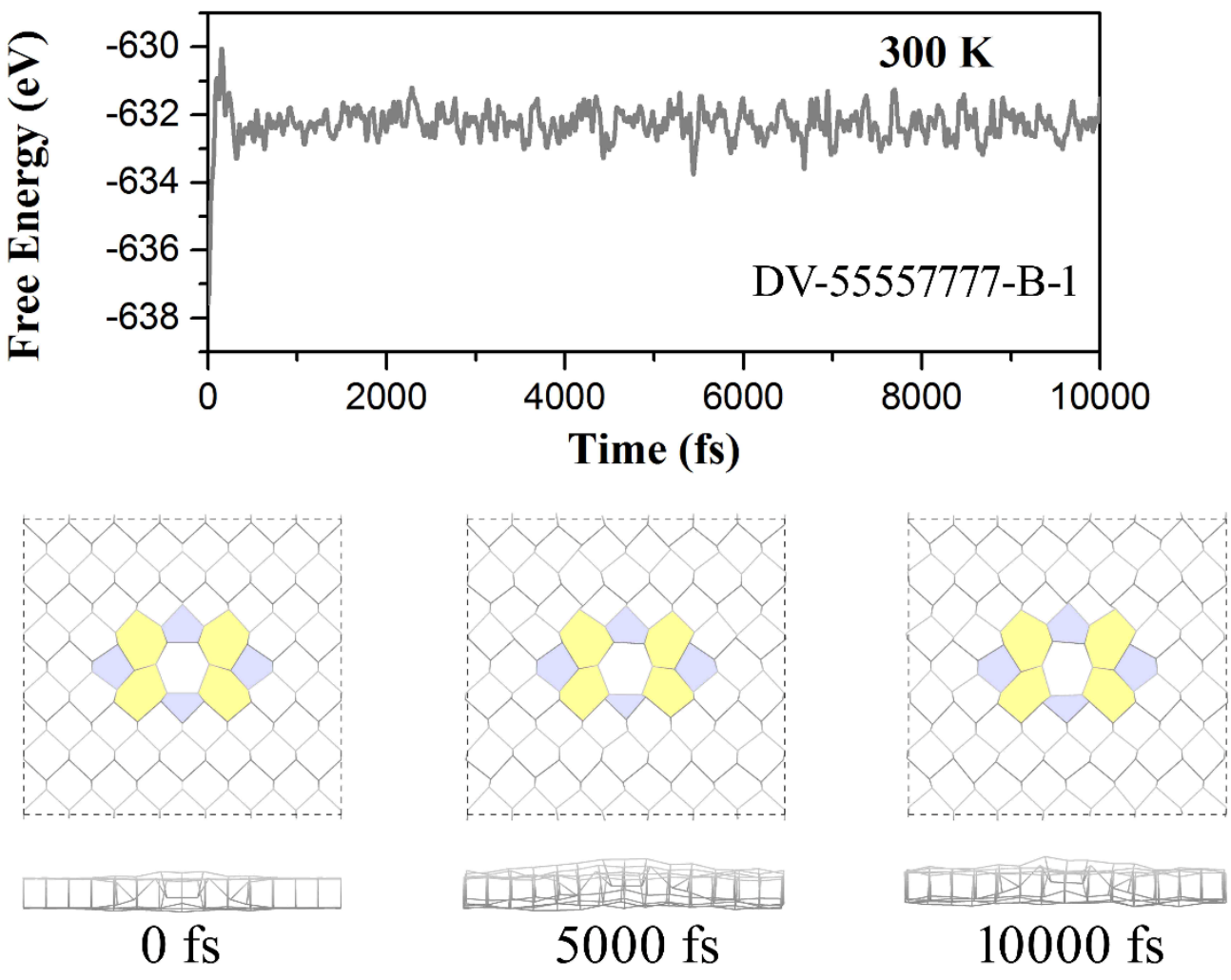
$5000 \mathrm{fs}$ $10000 \mathrm{fs}$

Figure S1 Variation of the free energy and snapshots of DV-55557777-B-1 in the AIMD. 

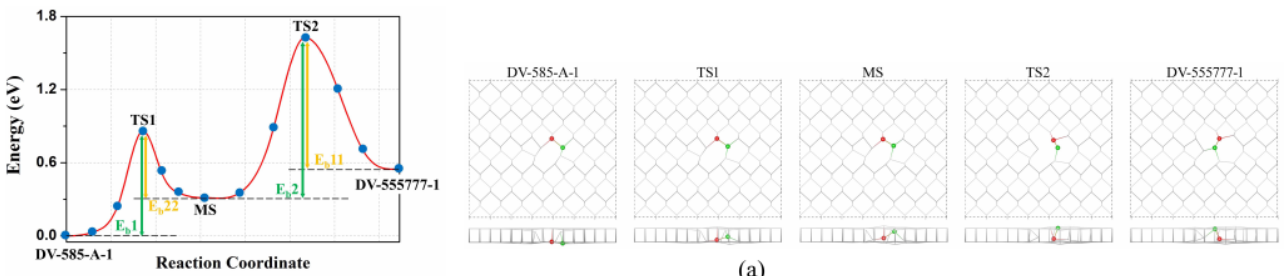

(a)
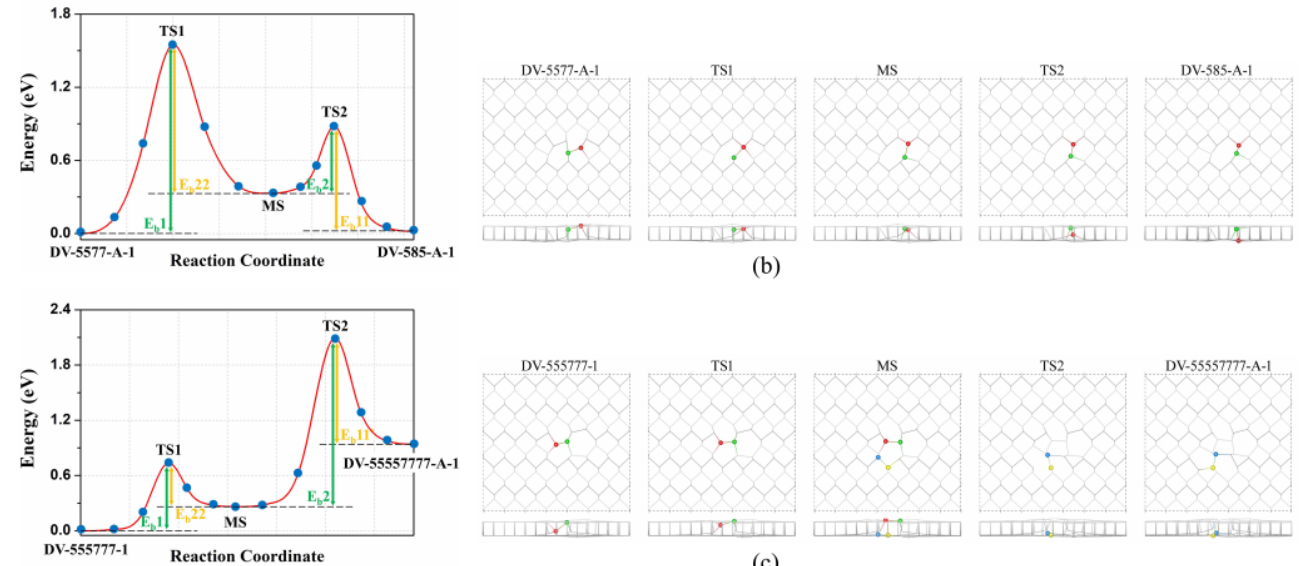

(c)
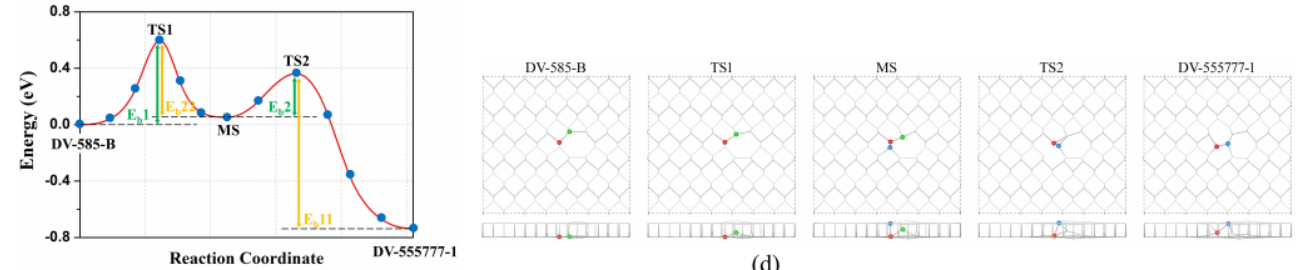

(d)
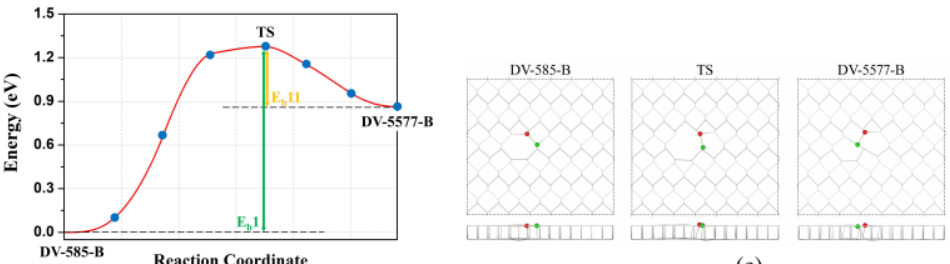

(e)
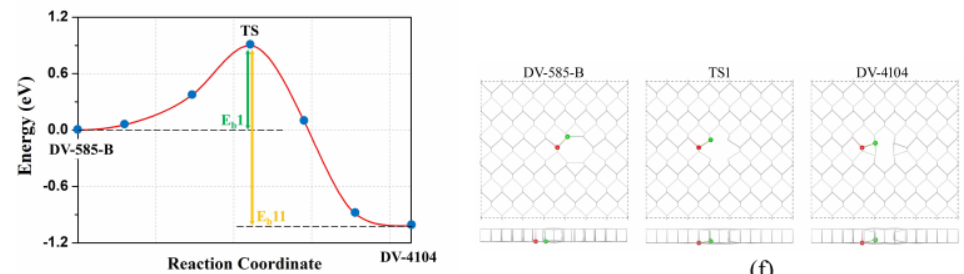

(f)

Figure S2 Minimum-energy paths (left) and associated atomic processes (right) for the transformations between different types of DVs in arsenene. (a) DV-585-A-1 $\leftrightarrow$ DV555777-1, (b) DV-5577-A-1 ↔ DV-585-A-1, (c) DV-555777-1 ↔ DV-55557777-A-1, (d) DV-585-B $\leftrightarrow$ DV-555777-1, (e) DV-585-B $\leftrightarrow$ DV-5577-B, and (f) DV-585-B $\leftrightarrow$ DV-4104. Dimers involved in Stone-Wales-like rotations are highlighted in color for clarify. 


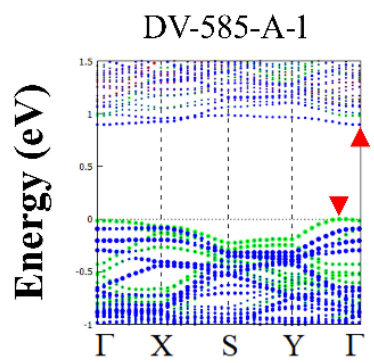

DV-555777-1

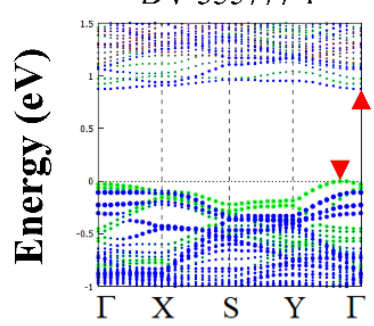

DV-5577-A-1

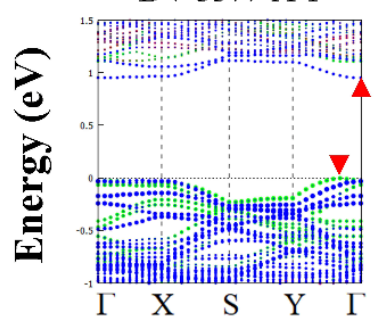

DV-55557777-A-1

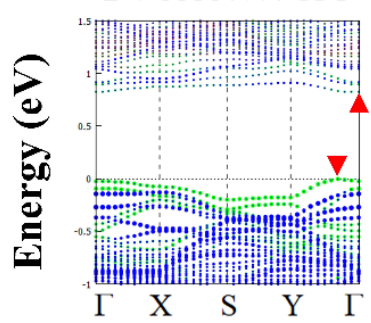

DV-585-A-2

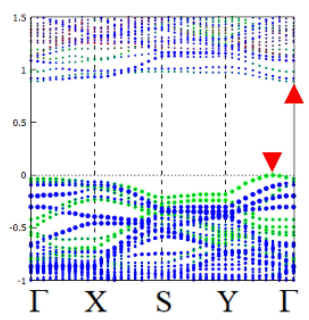

DV-555777-2

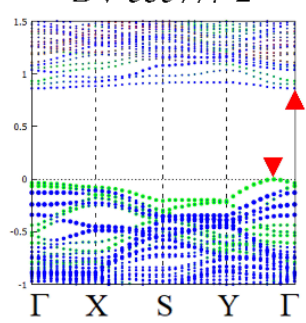

DV-5577-A-2

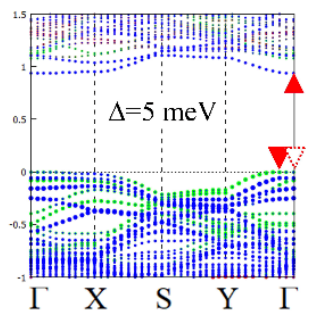

DV-55557777-A-2

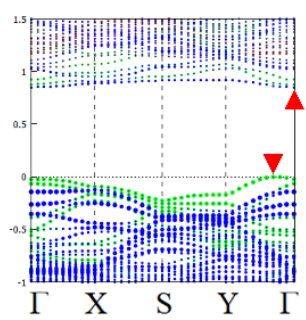

DV-585-A-3

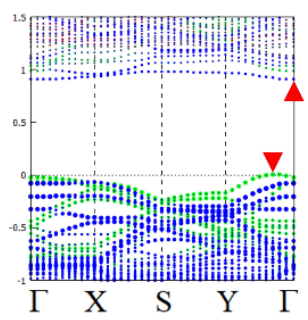

DV-585-B

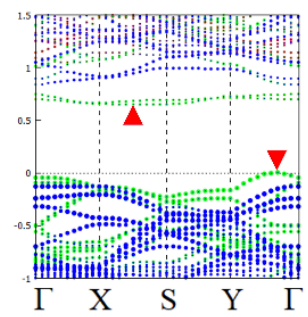

DV-4104

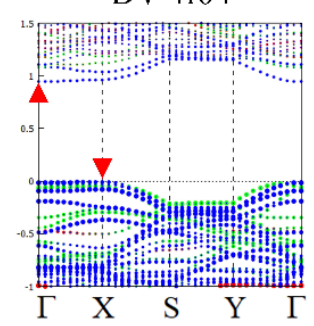

DV-5577-A-3

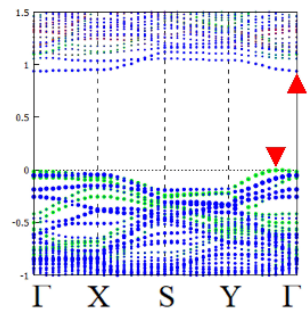

DV-5577-B

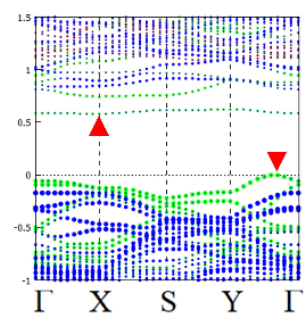

DV-55557777-A-4

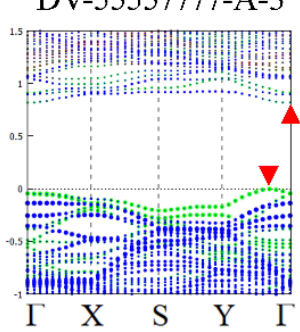

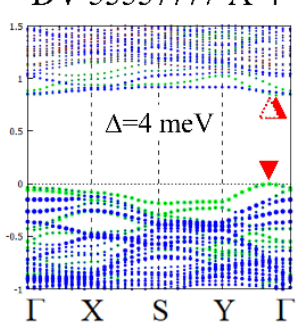

Figure S3 Orbital-resolved band structures of arsenene monolayers with DVs. The $p_{\mathrm{x}}$, $p_{\mathrm{y}}$, and $p_{\mathrm{z}}$ orbitals of the As atom are represented by the red, green, and blue dotted lines, respectively. The band edges are indicated by red hollow (associated with the almost direct band gap) and solid triangles. $\Delta$ is the energy difference between indirect and direct band gaps. Fermi levels are indicated by black dashed lines. 


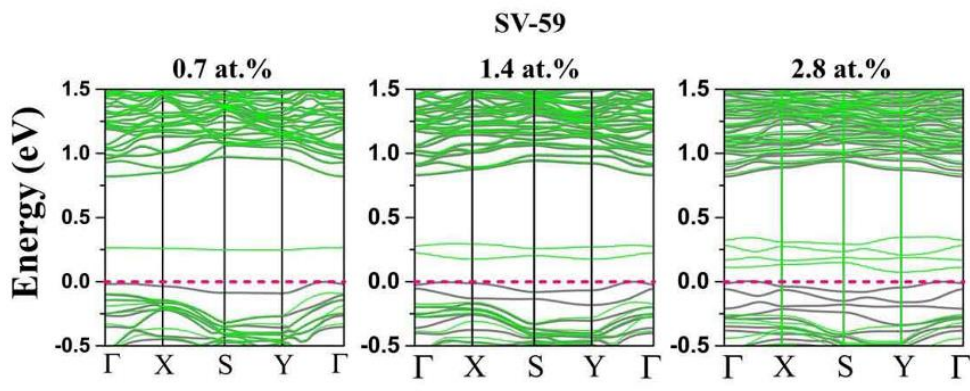

DV-55557777-B-1

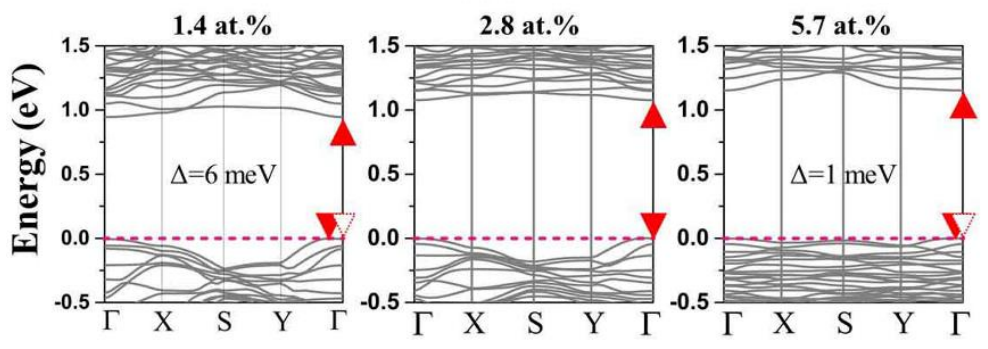

DV-55557777-B-2

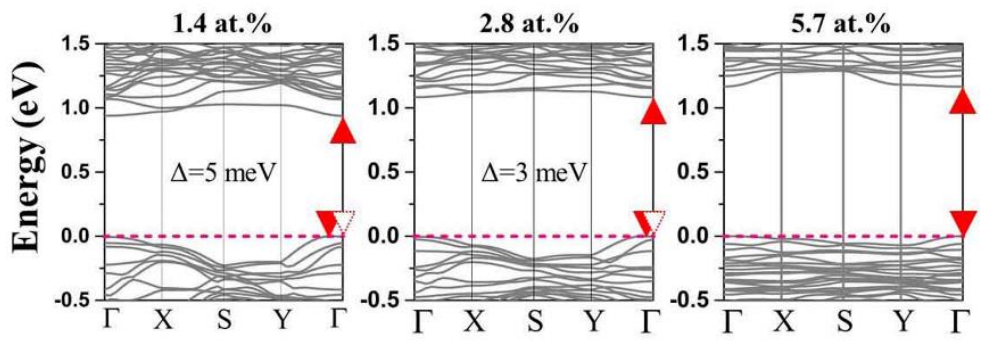

DV-55557777-B-3

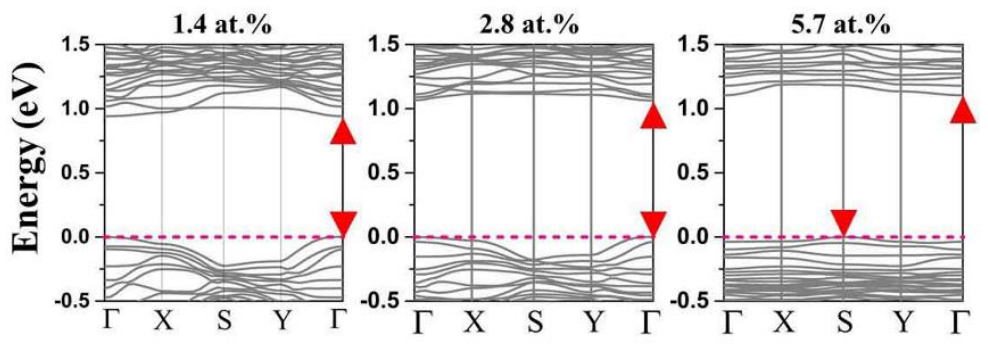

DV-55557777-B-4

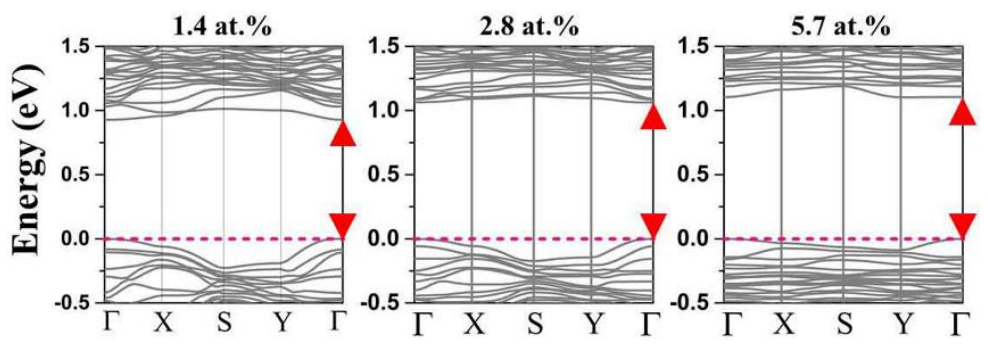

Figure S4 Defect concentration-dependent band structures of DV-55557777-Bdecorated arsenene monolayers. The band edges are indicated by red hollow (associated with the almost direct band gap) and solid triangles. $\Delta$ is the energy difference between indirect and direct band gaps. Fermi levels are indicated by red dashed lines. 